\title{
Biochemical characterization and zinc binding group (ZBGs) inhibition studies on the catalytic domain of MMP7 (cdMMP7)
}

Fan Meng, Hao Yang, Colin Jack, Huaqun Zhang, Abraham Moller, Devin Spivey, Richard C. Page,* David L. Tierney,* and Michael W. Crowder*

Department of Chemistry and Biochemistry Miami University, Oxford, Ohio, 45056, U.S.A

*To whom correspondence should be addressed:

Department of Chemistry and Biochemistry, Miami University, Oxford, Ohio 45056, U.S.A

Michael W. Crowder e-mail: crowdemw@miamioh.edu phone: 513-529-2813

David L. Tierney e-mail: tiernedl@miamioh.edu $\quad$ phone: 513-529-8234

Richard C. Page e-mail:_pagerc@miamioh.edu phone: 513-529-2281 


\begin{abstract}
Matrix metalloproteinase 7 (MMP7/matrilysin-1) has been implicated in many pathological conditions, such as in cancer and inflammatory diseases; therefore, MMP7 has been targeted for drugs. Success in developing a clinical inhibitor, which exhibits suitable specificity and selectivity, will likely require structural and/or kinetic evaluation of enzyme/inhibitor interactions. To enable these future studies we herein describe the over-expression, purification, and characterization of the catalytic domain of MMP7 (cdMMP7). cdMMP7 was over-expressed in an E. coli over-expression system, and the resulting enzyme was processed into inclusion bodies, which were subsequently solubilized, enabling the enzyme to be re-folded into a catalytically-active form. cdMMP7 was shown to bind 1.8 eq of $\mathrm{Zn}(\mathrm{II})$, exhibit steady-state kinetic constants of $0.4 \mathrm{~s}^{-1}$ for $k_{\text {cat }}$ and $23 \mu \mathrm{M}$ for $\mathrm{K}_{\mathrm{m}}$, and yield CD and fluorescence spectra that are consistent with a properly-folded enzyme. Pre-steady state kinetic studies yielded kinetic mechanisms of cdMMP7, and these mechanisms are similar to those of other MMPs. Inhibition studies on cdMMP7 with four zinc binding group (ZBG) inhibitors showed that maltol, thiomaltol, and allothiomaltol are better inhibitors with lower $\mathrm{IC}_{50}$ values and lower $\mathrm{K}_{\mathrm{d}}$ values against cdMMP7 and cdMMP16 than the commonly-used ZBG inhibitor acetohydroxamic acid. Docking studies suggest that improved inhibitory character may be due to interactions with the S1' substrate binding pocket. Finally, a ZnCo-heterobimetallic analog of cdMMP7 with $\mathrm{Co}(\mathrm{II})$ bound in the catalytic site was prepared and characterized. This study describes a wellcharacterized analog of MMP7 that is available for future inhibitor design efforts.
\end{abstract}




\section{Graphical abstract}

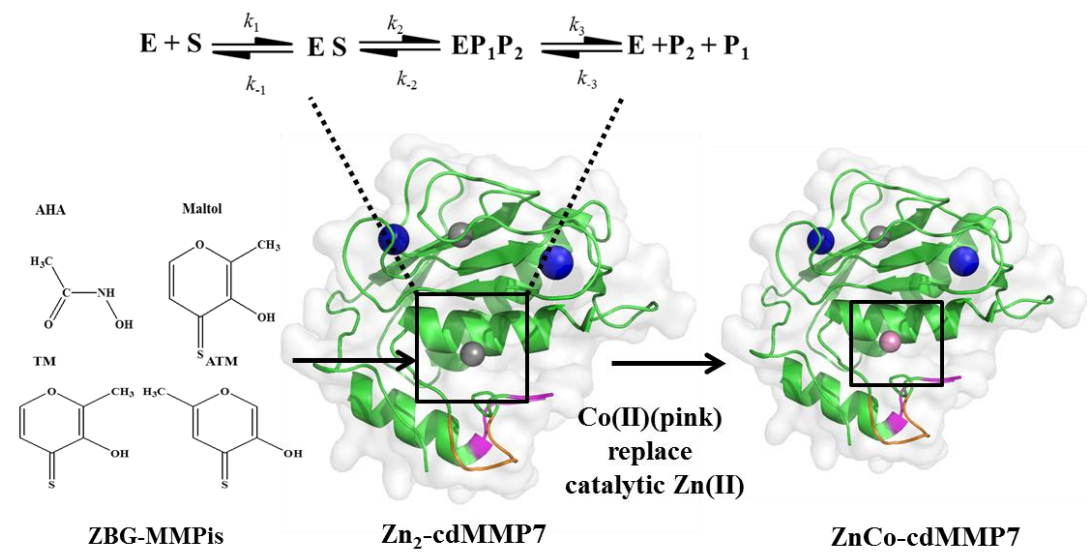

Kinetic studies to probe the kinetic mechanism of the catalytic domain of matrix metalloproteinase 7 (cdMMP7); preparation of Co(II)-substituted MMP7 for future spectroscopic studies; small molecule zinc binding group (ZBG) inhibition studies on cdMMP7

\section{Keywords}

Matrix metalloproteinases (MMPs), pre-steady-state kinetic study, cobalt(II)-substitution, zincbinding group MMP inhibitors (ZBG-MMPis)

\section{Author contributions}

MF, DT, RP, MWC conceived and coordinated the study and wrote the manuscript. CJ performed DNA cloning work and prepared the over-expression plasmid for cdMMP7. MF and DS prepared and characterized cdMMP7 and ZnCo-cdMMP7. MF and HY performed the stopped flow intrinsic fluorescence emission experiments and $\mathrm{ZBG}^{-\mathrm{IC}_{50}}$ assays on cdMMP7/16. MF performed the stopped flow substrate-hydrolysis experiments. AM calculated theoretical 
equations for $k_{\text {cat }}$ and $\mathrm{K}_{\mathrm{m}}$ by using the King-Altman method. MF and ZHQ performed the ITC experiments. RP performed computational docking studies.

\section{Funding}

This work was supported by the National Science Foundation (CHE-1509285 to MWC and DLT, and MCB-1552113 to RCP).

\section{Introduction}

Matrix metalloproteinases (MMPs) are Zn(II)-dependent metalloproteases, and there are 23 distinct MMPs in humans[1]. The MMPs can be broadly categorized into two classes: secreted MMPs and membrane-bound MMPs (MT-MMPs). Matrix metalloproteinase 7 (MMP7) is a member of the matrilysin subclass of secreted MMPs[1, 2]. Full-length MMP7 consists of two conserved functional domains: a $\mathrm{N}$-terminal pro-domain and a $\mathrm{C}$-terminal catalytic domain 
$[3,4]$. The function of the pro-domain is to maintain MMP7 in a latent form (proMMP7), and this latency is achieved by coordination of the active site $\mathrm{Zn}$ (II) with a cysteine residue, which is located in the highly-conserved PRCGPD "bait region" motif in the pro-domain[2, 5]. When proMMP7 is secreted into the extracellular space, the enzyme is cleaved by one of several serine or metazincin proteases, leading to the release of the pro-domain and activation of MMP7. This activation of MMP7, known as the "cysteine switch", is a common regulatory mechanism in secreted MMPs[5-7]. The catalytic domain has a ball-like structure consisting of three $\alpha$-helices, five $\beta$-sheets, and multiple loops.[8] The catalytic domain contains $2 \mathrm{Zn}(\mathrm{II})$ binding sites: a structural site with $\mathrm{Zn}(\mathrm{II})$ coordinated by 3 conserved histidines and an aspartic acid and a catalytic site with $\mathrm{Zn}(\mathrm{II})$ coordinated by 3 conserved histidines found in a conserved HExxHxxGxxH motif, and 1-2 solvent molecules (Figure 1)[8]. Adjacent to the active site are a set of substrate binding pockets, termed S1-S3 and S1'-S3'. The substrate binding pockets of MMPs vary in shape, charge, and hydrophobicity and are considered key factors in selectivity and specificity for substrates and inhibitors[8-10]. A conserved methionine, Met237, is present 8 amino acids from the catalytic Zn site as part of "Met-turn", which is conserved in all MMPs belonging to the metazincin family and is essential for stabilizing $\mathrm{Zn}$ (II)-binding motif for metal ion coordination $[8,11]$. MMP7 also contains a ten amino acid loop termed the specificity loop (Tyr241-Leu251) in the C-terminus, which is thought to contribute to selectivity in substrate binding [12] (Figure 1).

The physiological substrates for MMP7 are components of the extracellular matrix (ECM), such as proteoglycan, fibronectin, casein, and elastin[13, 14]. Studies have shown that MMP7 affects cell-ECM adhesion and cell-cell interactions, which allows for matrix remodeling, cellular motility, cellular growth, cellular proliferation, and angiogenesis[13, 15]. In addition to 
its roles in regulation of ECM biology, MMP7 has been implicated in mediating multiple signaling pathways (immune response, inflammation, and neurotransmitter processing) by processing key molecules.[13, 14, 16-21]. Up-regulation of MMP7 has been observed in tumorigenesis of breast, colon, and pancreatic cancers and in arthritis and ulcers [13, 22-28]. Through these multitude of targets and role in myriad biological processes and pathways, MMP7 is considered to be a potential therapeutic target[29]. Previous attempts to use broad-spectrum MMP inhibitors (MMPis) in clinical trials have failed, due primarily to reported severe side effects like musculoskeletal syndrome (MSS). In studies with inhibitors targeting MMP7, “offtarget" inhibition of other MMPs (and perhaps other proteases) was used to explain the failure of the inhibitors clinically[10, 30, 31]. Therefore, improved selectivity and specificity are required if MMPis are to be used as effective and safe therapeutics[30, 32].

Due to the highly similar catalytic domains of MMPs (cdMMPs), particularly at the active sites, the development of a high specificity MMPi has been challenging[9]. One common approach to improve MMPi selectivity and efficacy is to optimize the interactions of the MMPi with adjacent substrate binding pockets, most notably the $S 1$ ' pocket[10, 33]. The architecture of S1' pockets vary significantly across MMPs; therefore, the S1' pocket is considered to be the most important site influencing substrate and inhibitor selectivity of the MMPs[34]. Unlike other MMPs with S1' pockets that are deep and have wide openings[10, 35, 36], MMP7 has a S1' pocket that is shallow with a narrow opening[8].

Cohen et al. reported structure-activity relationship (SAR) studies with hydroxamatecontaining zinc binding groups (ZBGs) containing substituents targeting the S1' pocket. These (SAR) studies showed that inhibitor selectivity can be achieved by targeting the S1' pocket in MMP3[16]. By analogy, we hypothesized that selective MMP7 inhibitors can be achieved by 
targeting the S1' pocket in MMP7. To conduct these studies, a well-characterized analog of MMP7 is needed. Herein, we report the over-expression, purification, and characterization of the catalytic domain of MMP7 (cdMMP7). We also report the preparation and characterization of Co(II)-substituted analogs of cdMMP7 for future spectroscopic interrogation of MMP7-inhibitor complexes. 


\section{Material and methods}

\subsection{Materials}

Taq PCR reaction kit, restriction enzymes (NdeI, HindIII), and T4 ligation kit were purchased from New England Biolabs (Ipswich, MA). Luria-Bertani (LB) medium was obtained from Invitrogen (Carlsbad, CA). Isopropyl- $\beta$ - $D$-thiogalactoside (IPTG) was procured from Anatrace (Maumee, OH). Tris(hydroxymethyl)aminomethane (Tris), 4-(2-hydroxyethyl)-1piperazineethanesulfonic acid (Hepes), urea, $\mathrm{NaCl}, \mathrm{CaCl}_{2}, \mathrm{ZnCl}_{2}, \mathrm{CoCl}_{2}$, ethylenediaminetetraacetic acid (EDTA), Brij35, Triton X100, sucrose, phenanthroline (OP), $\mathrm{D}_{2} \mathrm{O}$, tris(2-carboxyethyl)phosphine (TCEP), arginine, and glutamate were purchased from Fisher Scientific (Hampton, New Hampshire). Barnstead NANOPure water was used to make all buffers. Chelex100 resin was procured from Biorad (Hercules, CA). Centricon ultrafiltration units were purchased from EMD Millipore (Billerica, MA), and Amicon centrifuge units (YM10 membranes) were obtained from Sigma Aldrich (St. Louis, MO). Fluorogenic peptide FS-6 (MCA-Lys-Pro-Leu-Gly-Leu-DNP-Dpa-Ala-Arg- $\mathrm{NH}_{2}$ Figure 2A) was purchased from Sigma Aldrich (St. Louis, MO) for pre-steady state kinetic, steady state kinetic, and inhibition studies. Fluorogenic peptide BML-P131 (Dnp-Pro-Leu-Gly-Leu-Trp-Ala-Arg- $\mathrm{NH}_{2}$, Figure 2B) and reference peptide for FS-6 (Mca-Pro-Leu-OH) were purchased from Enzo Life Science (Farmingdale, NY). Acetohydroxamic acid (AHA) and maltol were procured from Sigma Aldrich. Thiomaltol (TM) and allothiomaltol (ATM) were kindly provided by Dr. Seth Cohen (University of California at San Diego, CA)

2.2. Cloning, over-expression, refolding, and purification of cdMMP7. A pGEM7-based plasmid, containing the cDNA for full-length MMP7, was generously provided by Dr. Carole L. 
Wilson (University of Washington). The gene for the catalytic domain of MMP7 (cdMMP7, corresponding to amino acids 100 to 271) was subcloned by PCR using the primers, 5'-aaa aaa cat atg tac tca cta ttt cca-3' and 5'-aaa aaa aag ctt cta ttt ctt tct tga-3'. These primers introduced NdeI and HindIII restriction sites at the 5' and 3' ends of the resulting gene, respectively. The PCR product was then digested and ligated into pET26b to yield pET26b/cdMMP7. DNA sequencing was used to confirm the cdMMP7 gene, and the over-expression plasmid was transformed into E. coli BL21(DE3) cells. A single colony was used to prepare a $50 \mathrm{~mL}$ overnight culture in Luria-Bertani (LB) medium containing $25 \mu \mathrm{g} / \mathrm{mL}$ of kanamycin (kan). The overnight culture was used to inoculate 4 X1 L of LB-kan (1:100 dilution), and the cultures were shaken at $37{ }^{\circ} \mathrm{C}$ with a $180 \mathrm{rpm}$ shaking rate until the cultures reached an optical density at 600 $\mathrm{nm}$ of 0.7 . Protein production was induced by adding isopropyl- $\beta-D$-thiogalactoside (IPTG) to 1 $\mathrm{mM}$ final concentration followed by shaking for 4 hours at $37{ }^{\circ} \mathrm{C}$. Cells were collected by centrifugation at $14,000 \mathrm{rpm}$ for 20 minutes at $4{ }^{\circ} \mathrm{C}$ and resuspended in lysis buffer (50 mM Tris, $\mathrm{pH}$ 7.5, containing $200 \mathrm{mM} \mathrm{NaCl}, 10 \%$ sucrose, and $1 \mathrm{mM}$ EDTA) containing $0.5 \mathrm{mM}$ lysozyme. Resuspended cells were ruptured by passing the suspension through a French press with a pressure of 1,500 psi three to four times, and the lysed mixture was centrifuged at 15,000 rpm for 25 minutes at $4{ }^{\circ} \mathrm{C}$. The resulting pellet, which contained cell debris and inclusion bodies, was washed three times with $30 \mathrm{~mL}$ of lysis buffer containing $0.1 \%$ Triton X100. Inclusion bodies were dissolved in $35 \mathrm{~mL}$ of $50 \mathrm{mM}$ Tris, $\mathrm{pH} 7.5$, containing $8 \mathrm{M}$ urea at room temperature for at least one hour with gentle stirring. Solubilized inclusion bodies were centrifuged at $4{ }^{\circ} \mathrm{C}$ for 10 minutes at $14,000 \mathrm{rpm}$ and then diluted to a final concentration of 0.3 $\mathrm{mg} / \mathrm{mL}$ with $50 \mathrm{mM}$ Tris, $\mathrm{pH} 7.5$, containing $6 \mathrm{M}$ urea. The concentration of dissolved inclusion bodies was determined by using a Pierce protein assay[37]. The diluted inclusion bodies 
(approximately $100 \mathrm{ml}$ ) were dialyzed twice against 2L $50 \mathrm{mM}$ Tris, $\mathrm{pH} 7.5$, containing $5 \mathrm{mM}$ $\mathrm{CaCl}_{2}, 100 \mu \mathrm{M} \mathrm{ZnCl}_{2}$, and $0.1 \%$ Brij35. Each dialysis step lasted 3-4 hours at $4{ }^{\circ} \mathrm{C}$, and a final dialysis step was allowed to proceed for approximately 12 hours, using $50 \mathrm{mM}$ Tris, $\mathrm{pH} 7.5$, containing $20 \mu \mathrm{M} \mathrm{ZnCl}_{2}, 5 \mathrm{mM} \mathrm{CaCl}_{2}$, and $0.1 \%$ Brij35. If refolded protein was prepared for isothermal titration calorimetry, inductively-couple plasma analysis or Co(II)-substitution an additional dialysis step was required against $50 \mathrm{mM}$ Tris, $\mathrm{pH} 7.5$, containing $5 \mathrm{mM} \mathrm{CaCl}_{2}, 1 \mu \mathrm{M}$ $\mathrm{ZnCl}_{2}$, and $0.1 \%$ Brij35 for 4 hours,. The purity of refolded protein was determined by SDSPAGE and MALDI-TOF mass spectrometry. The concentration of purified cdMMP7 was determined by measuring the absorbance at $280 \mathrm{~nm}$ and using a calculated extinction coefficient of $30,000 \mathrm{M}^{-1} \mathrm{~cm}^{-1}[38]$.

2.3. Metal analyses of cdMMP7 analogs. The metal content of cdMMP7 samples was determined using a Perkin Elmer Optima 7300DV Inductively-Coupled Plasma with Atomic Emission Spectrometer. The emission wavelengths were set to $213.8 \mathrm{~nm}$ and $238.9 \mathrm{~nm}$ for measuring zinc and cobalt, respectively, as previously described[39, 40].

2.4. Steady state kinetic studies on cdMMP7. Steady-state kinetic studies were conducted on cdMMP7 using a Synergy HT plate reader. The fluorescent substrate FS6 (Sigma Aldrich) was used in the assay, and excitation and emission wavelengths were set to $330 \mathrm{~nm}$ and $420 \mathrm{~nm}$, respectively. The concentration of substrate was varied between 10 and $60 \mu \mathrm{M}$, and the concentration of $\mathrm{Zn}_{2}$-cdMMP7 (analog of cdMMP7 with $\mathrm{Zn}$ (II) in the catalytic and structural sites) was fixed to $10 \mathrm{nM}$ (or to $20 \mathrm{nM}$ with $\mathrm{ZnCo}$-cdMMP7). The final volume for each reaction 
was $100 \mu \mathrm{L}$, and each reaction was allowed to proceed for 5 minutes. GraphPad Prism 5 was used to fit the resulting kinetic data to the Michaelis equation to obtain $\mathrm{K}_{\mathrm{m}}$ and $k_{\mathrm{cat}}[41,42]$.

\subsection{Stopped-flow kinetic studies on cdMMP7 and cdMMP16. An Applied Photophysics} spectrophotometer was used to conduct stopped-flow fluorescence studies. The excitation wavelength was set to $280 \mathrm{~nm}$. Two different fluorescence cut-off filters were used depending on whether intrinsic tryptophan fluorescence or substrate fluorescence was monitored over time. In experiments monitoring intrinsic tryptophan fluorescence, a $320 \mathrm{~nm}$ cut-off filter was used to exclude all emission wavelengths under $320 \mathrm{~nm}$. In these experiments at $25^{\circ} \mathrm{C}$, the concentration of cdMMP7 was fixed at $10 \mu \mathrm{M}$, and BML-P131 was used as the substrate (concentrations varied between 12 and $100 \mu \mathrm{M}$ ). The reaction buffer was $50 \mathrm{mM}$ Hepes, $\mathrm{pH}$ 7.0, containing 100 $\mathrm{mM} \mathrm{NaCl}$ and $5 \mathrm{mM} \mathrm{CaCl}_{2}$. The reaction was monitored over 20 seconds using a $125 \mu$ s time interval. Dynafit 3.0 was used to simulate progress curves to obtain microscopic rate constants $\left(k_{1}, k_{-1}\right.$, and $\left.k_{2}\right)$.

In reactions in which the fluorescence of substrate was monitored, a $400 \mathrm{~nm}$ cutoff filter was used to filter out emission wavelengths below $400 \mathrm{~mm}$, including fluorescence emission of protein tryptophans. The concentrations of cdMMP7 and cdMMP16 samples were $0.5 \mu \mathrm{M}$, and the buffer was $50 \mathrm{mM}$ Hepes, $\mathrm{pH} 7.5$, containing $100 \mathrm{mM} \mathrm{NaCl}$ and $5 \mathrm{mM} \mathrm{CaCl}$. The final concentrations of substrate FS-6 were varied between $70 \mu \mathrm{M}$ and $4.9 \mu \mathrm{M}$ for studies with cdMMP7 and $32 \mu \mathrm{M}$ to $1.98 \mu \mathrm{M}$ for studies with cdMMP16. After mixing, emission intensities were obtained every $125 \mu$ s for 1 second. The resulting progress curves were simulated to a standard uni-bi hydrolytic mechanism[43] by using Dynafit 3.0 to obtain microscopic rate constants[44]. The King-Altman method was used to generate theoretical expressions for $k_{\text {cat }}$ and $\mathrm{K}_{\mathrm{m}}[45]$, and the theoretically-calculated steady-state kinetic constants were compared to those 
kinetic constants determined experimentally. The theoretical expressions were $k_{\mathrm{cat}}=\left[k_{2}\left(k_{3}+k_{-2}\right)\right] /$ $\left(k_{3}+k_{-2}+k_{2}\right)$ and $\left.\mathrm{K}_{\mathrm{m}}=\left(k_{2} k_{3}+k_{-2} k_{-1}+k_{3} k_{-1}\right) /\left(k_{3} k_{1}+k_{1} k_{-2}+k_{1} k_{2}\right)\right]$.

2.6. Preparation of the ZnCo-cdMMP7 analog. An analog of cdMMP7 that contains $\sim 1$ equivalent of $\mathrm{Zn}(\mathrm{II})$ was prepared using a previously-published procedure[46]. Briefly, purified cdMMP7 was diluted to $\sim 100 \mu \mathrm{M}$ in a total volume of $\sim 5 \mathrm{~mL}$. The sample was dialyzed against three changes of $1 \mathrm{~L}$ of $20 \mathrm{mM}$ Hepes, $\mathrm{pH} 7.0$, containing $2 \mathrm{mM}$ 1,10-phenanthroline (OP) and 5 $\mathrm{mM} \mathrm{CaCl} 2$ at $4{ }^{\circ} \mathrm{C}$. The dialysis buffer was changed every 3 hours. The sample was then dialyzed against $2 \mathrm{X} 1 \mathrm{~L}$ of Chelex100-treated, $20 \mathrm{mM}$ Hepes, $\mathrm{pH} 7.0$, containing $5 \mathrm{mM} \mathrm{CaCl}_{2}$ for $(3$ hours for each step). The resulting sample was made $1 \mathrm{mM}$ in $\mathrm{CoCl}_{2}$, allowed to incubate for at least 40 minutes on ice, and dialyzed for 10 hours versus $1 \mathrm{~L}$ of $20 \mathrm{mM}$ Hepes, $\mathrm{pH}$ 7.0, containing $1 \mathrm{mM}$ $\mathrm{CoCl}_{2}$ and $5 \mathrm{mM} \mathrm{CaCl}_{2}$ and twice against 1L of Chelex100-treated, $20 \mathrm{mM}$ Hepes, $\mathrm{pH}$ 7.0, containing $5 \mathrm{mM} \mathrm{CaCl}_{2}$ (3 hours each step). Finally, the sample was dialyzed versus $1 \mathrm{~L}$ of 50 $\mathrm{mM}$ Hepes, $\mathrm{pH} 7.5$, containing $100 \mathrm{mM} \mathrm{NaCl}, 5 \mathrm{mM} \mathrm{CaCl}_{2}$, and $0.4 \mathrm{mM}$ TCEP for 4 hours before the sample was concentrated using a Centricon unit to a final concentration of approximately $100 \mu \mathrm{M}$.

2.7. Circular dichroism and fluorescence emission spectroscopic studies of cdMMP7. cdMMP7 was diluted to $10 \mu \mathrm{M}$ with $20 \mathrm{mM}$ sodium phosphate, $\mathrm{pH} 7.0$, containing $100 \mathrm{mM}$ $\mathrm{NaCl}$. CD spectra were obtained on a JASCO J-810 CD spectropolarimeter using a $1 \mathrm{~cm}$ cylindrical cell. The spectra were recorded in the range of $190-260 \mathrm{~nm}$, and 10 scans were signal-averaged at $25{ }^{\circ} \mathrm{C}$. DiChroWeb was used to estimate the secondary structure 
composition[47]. Fluorescence emission spectra were collected on a Perkin Elmer Luminescence spectrometer (Model LS-55), using an excitation wavelength of $280 \mathrm{~nm}$. Emission spectra from 290 to $500 \mathrm{~nm}$ were signal-averaged (5 scans) at room temperature.

2.8. Optical spectroscopy of ZnCo-cdMMP7. UV-Vis spectra on cdMMP7 samples were collected on a Lambda $850 \mathrm{UV}$-vis spectrophotometer from $200-800 \mathrm{~nm}$, operating at approximately $25{ }^{\circ} \mathrm{C}$. ZnCo-cdMMP7 samples were concentrated to $100 \mu \mathrm{M}$ using a Centricon, and the buffer was $50 \mathrm{mM}$ Hepes, $\mathrm{pH} 7.0$, containing $100 \mathrm{mM} \mathrm{NaCl}$ and $5 \mathrm{mM} \mathrm{CaCl}_{2}$. Difference spectra were obtained by subtracting the spectrum of metal-free cdMMP7 from those of the ZnCo-cdMMP7 analogs.

2.9. Determination of $I C_{50}$ values for ZBGs against cdMMP7 and cdMMP16. Four zinc binding group (ZBG) inhibitors[48] were used in inhibitor studies with cdMMP16 and cdMMP7: acetohydroxamic acid (AHA), maltol, thiomaltol (TM), and allothiomaltol (ATM). Steady-state kinetic assays for determining $\mathrm{IC}_{50}$ were conducted on a Synergy HT plate reader (BioTEK). The concentration of the cdMMPs was $10 \mathrm{nM}$, and the concentration of substrate FS-6 was $10 \mu \mathrm{M}$. The reaction volume was $100 \mu \mathrm{L}$, and the buffer was $20 \mathrm{mM}$ Hepes, $\mathrm{pH}$ 7.0, containing $100 \mathrm{mM}$ $\mathrm{NaCl}$ and $5 \mathrm{mM} \mathrm{CaCl}_{2}$. The cdMMP samples were incubated with various concentrations of the ZBGs at room temperature for no more than 30 minutes, and each reaction was monitored for 10 minutes. The concentrations of AHA, maltol, TM, and ATM were varied between 0 - $1.2 \mathrm{M}, 0$ $620 \mathrm{mM}, 0-12.3 \mathrm{mM}$, and $0-12.3 \mathrm{mM}$, respectively. The values for $\mathrm{IC}_{50}$ were calculated by using GraphPad Prism 5. 
2.10. Isothermal titration calorimetry (ITC) on cdMMP 16 and cdMMP7. The binding of ZBGs to the cdMMPs was monitored using a NanoITC (TA Instruments) isothermal titration calorimeter. Samples of cdMMP16 and cdMMP7 were diluted to concentrations of $50 \mu \mathrm{M}$ using $50 \mathrm{mM}$ Hepes, $\mathrm{pH}$ 7.5, containing $100 \mathrm{mM} \mathrm{NaCl}$ and $5 \mathrm{mM} \mathrm{CaCl}_{2}$ for cdMMP16 and $50 \mathrm{mM}$ Tris, $\mathrm{pH} 7.5$, containing $5 \mathrm{mM} \mathrm{ZnCl}_{2}$, and $0.1 \%(\mathrm{w} / \mathrm{v})$ Brij35 for cdMMP7. Inhibitors were diluted with the same buffers used to dilute the enzyme to minimize any heat of dilution effects[49]. Concentrations of ATM and TM stocks were $500 \mu \mathrm{M}$ and $350 \mu \mathrm{M}$, respectively. The enzymes were titrated with $1.9 \mu \mathrm{L}$ ligand per injection for a total of 24 injections. The NanoITC program (TA Instruments) was used to fit the experimental data to yield values for change in enthalpy $(\Delta \mathbf{H})$, change in entropy $(\Delta \mathbf{S})$, dissociation constant $\left(\mathbf{K}_{\mathbf{d}}\right)$, and binding stoichiometry (n). The change in Gibbs free energy $(\boldsymbol{\Delta G})$ for the binding of each ZBG was calculated using $\Delta \mathrm{G}=\Delta \mathrm{H}-\mathrm{T} \Delta \mathrm{S}$.

2.11. ZBG docking studies. Models of cdMMP7 and cdMMP16 were prepared from PDB depositions $1 \mathrm{mmp}$ and $1 \mathrm{rm} 8,[8,50]$ respectively, using the "prepack" protocol within the Rosetta modeling software[51]. Rosetta ligand parameter files were prepared from the final geometries and charges for conformers of thiomaltol or allothiomaltol generated using OMEGA[52]. Ligands placed in a starting pose near the catalytic zinc site were paired with prepacked cdMMP7 and cdMMP16 coordinates for RosettaLigand docking calculations[53, 54]. Docking runs allowed for backbone and side-chain flexibility within cdMMP7 or cdMMP16 with extra Chi1 and aromatic-Chi2 rotamers and permitted ligand translations away from the starting pose 
of up to $\pm 5 \AA$ along the $\mathrm{x}, \mathrm{y}$ and $\mathrm{z}$ axes. An ensemble of 10,000 decoys was generated for each docking calculation, and the top 50 were clustered based upon overall Rosetta energy score (total_score). A representative model for MMP/ZBG pair was selected from the low energy ensemble. 


\section{Results}

3.1. Over-expression, refolding, and purification of cdMMP7. In order to obtain large quantities of catalytically-active, recombinant cdMMP7, we used a pET26b-based vector containing the gene for cdMMP7, and we over-expressed the enzyme in BL21(DE3) E. coli cells, in a method similar to that of Cha et al.[46]. Recombinant cdMMP7 was processed into inclusion bodies after over-expression in E. coli. Previously, Cha et al. solubilized the inclusion bodies in 6 M Gdn$\mathrm{HCl}$ and removed the detergent using a single dialysis step with buffer containing $0.05 \%$ Brij35, $10 \mathrm{mM} \mathrm{CaCl}$, and $20 \mu \mathrm{M} \mathrm{ZnCl}_{2}$.[46] The resulting protein was purified using a hemopexinaffinity column, and fractions containing cdMMP7 were identified by activity assays[46]. Our initial efforts at purifying cdMMP7 used a modified Cha method[46], in which we replaced Gdn$\mathrm{HCl}$ with urea. In our hands, we obtained low levels of cdMMP7 when using urea, and SDSPAGE showed that the enzyme was hydrolyzed (Figure S1A).

Therefore, we solubilized the inclusion bodies with $8 \mathrm{M}$ urea, and the enzyme was folded by slow removal of urea using dialysis. Three significant changes in the dialysis steps were used: (1) the concentration of Brij35 was increased from $0.05 \%(w / v)$ to $0.10 \%(w / v)[55]$ to increase the solubility of refolding intermediates during dialysis, (2) the single dialysis step used in the Cha method[46] was replaced by three shorter steps (each dialysis step was 3 to 4 hours besides the final step), (3) the concentration of $\mathrm{ZnCl}_{2}$ was $100 \mu \mathrm{M}$ in the first two dialysis steps, $20 \mu \mathrm{M}$ in the third step, and the resulting cdMMP7 was shown to be intact and pure by SDS-PAGE (Figure S1B). The yield of cdMMP7 was $~ 2.4 \mathrm{mg}$ per liter of LB growth medium. ICP-AES showed that the recombinant enzyme binds $1.8 \pm 0.1$ equivalents of $\mathrm{Zn}(\mathrm{II})$ (Table 1). Steadystate kinetic studies using FS- 6 as the substrate yielded a $k_{\text {cat }}$ of $0.40 \pm 0.02 \mathrm{~s}^{-1}$ and a $\mathrm{K}_{\mathrm{m}}$ of $23 \pm$ $2 \mu \mathrm{M}$ (Table 1). CD spectra of the recombinant enzyme (denoted as $\mathrm{Zn}_{2}$-cdMMP7) showed $27 \%$ 
$\alpha$-helix and 33\% $\beta$-sheet (Figure S2 and Table S1). The fluorescence spectrum of the refolded cdMMP7 showed a single emission peak centered at 340 nm (Figure 3A)

The initial concentration $(\leq 0.3 \mathrm{mg} / \mathrm{mL})$ of inclusion bodies was critical in obtaining properly-folded, catalytically-active cdMMP7. If high concentrations $(>0.5 \mathrm{mg} / \mathrm{ml})$ of solubilized cdMMP7 inclusion bodies were used, the resulting enzyme (called inactive $\mathrm{Zn}_{2}$-cdMMP7) exhibited a slightly shifted CD spectrum (Figure S2), lower metal to protein ratios ( 1.5 Zn(II) equivalents), a significantly-altered fluorescence spectrum that had two emission peaks (Figure S3), and no catalytic activity.

3.2. Stopped-flow fluorescence studies. Previously, we reported that the fluorescence emission properties of presumably highly-conserved Trp154 in cdMMP1 and cdMMP16 changed during the hydrolysis of substrate BML-P131 (Figure 2B). Trp154 is approximately $10 \AA$ away from the catalytic Zn(II) in MMP1, MMP7, and MMP16[39, 40]. Therefore, we conducted stopped-flow fluorescence studies with cdMMP7 using BML-P131 as substrate (Figure 3B). BML-P131 is hydrolyzed slowly by cdMMP7, so we followed the reaction for 20 seconds. During the first 5 seconds of the reaction, there was a decrease in fluorescence emission, followed by an increase in fluorescence over the subsequent 15 seconds. The progress curves were fitted to a simple kinetic mechanism[44] (Table 2), and we were able to obtain microscopic rate constants $\left(k_{1}, k_{-1}\right.$, and $k_{2}$ ) for the hydrolysis of BML-P131by cdMMP7. These microscopic rate constants are much lower than those for MMP1 and MMP16[39, 40], demonstrating that BML-P131 is a poor substrate for cdMMP7 (Table 2). The rate of fluorescence decay (first phase of progress curve in Figure 3B) was plotted against the substrate concentration as previously reported[39], and the $\mathrm{K}_{\mathrm{s}}$ for BML-P131 for cdMMP7 is $30.5 \mu \mathrm{M}$ (Table 2). 
In an effort to further probe the reaction mechanism of the MMPs, we conducted stopped-flow fluorescence studies on cdMMP7 (Figure 3C) and cdMMP16 (Figure 3D) with fluorescent substrate FS-6. The progress curves for both cdMMPs were similar, exhibiting a burst phase during the initial $0.2 \mathrm{sec}$ of the reaction for cdMMP7 and during the initial $0.1 \mathrm{sec}$ of the reaction for cdMMP16 (Figure $3 \mathrm{C} \& \mathrm{D}$ ). The burst, which had an amplitude that was substrate-concentration dependent, was followed by a relatively slower phase. The stopped-flow progress curves were fitted using Dynafit to a uni-bi hydrolytic mechanism[43] (see lines in Figure $3 \mathrm{C} \& \mathrm{D})$. The three-step kinetic mechanism proposed for cdMMP7 and cdMMP16 is shown in Table 3. The best fit to the data for both MMPs assumed rate-limiting chemistry steps $\left(k_{2}\right)$ and slow reverse chemistry steps $\left(k_{-2}\right)$ (Table 3$)$. To test the validity of our proposed kinetic mechanism, we used the King-Altman method to calculate the theoretical expressions for $k_{\text {cat }}$ and $\mathrm{K}_{\mathrm{m}}$. The theoretical $k_{\text {cat }}$ values for cdMMP7 and cdMMP16 were $0.58 \mathrm{~s}^{-1}$ and $1.6 \mathrm{~s}^{-1}$, and the theoretical $\mathrm{K}_{\mathrm{m}}$ values were $27 \mu \mathrm{M}$ and $13 \mu \mathrm{M}$, respectively. These theoretical steady-state kinetic constants are similar to those determined experimentally: cdMMP7 has a $k_{\text {cat }}$ of $0.40 \mathrm{~s}^{-1}$ and a $\mathrm{K}_{\mathrm{m}}$ of $23 \mu \mathrm{M}$ (Table 1) and cdMMP16 has a $k_{\text {cat }}$ of $1.1 \mathrm{~s}^{-1}$ and a $\mathrm{K}_{\mathrm{m}}$ of $10.6 \mu \mathrm{M}[39]$.

3.3. Preparation and characterization of heterobimetallic ZnCo-cdMMP7. Using a previouslypublished procedure[46], we prepared an analog of cdMMP7, which contains $\sim 1$ equivalent of $\mathrm{Zn}$ (II). The addition of Co(II) to this sample resulted in a $\mathrm{ZnCo-cdMMP7} \mathrm{analog} \mathrm{that} \mathrm{binds} 0.7$ eq of $\mathrm{Zn}(\mathrm{II})$ and 1.2 eq of $\mathrm{Co}$ and exhibits a $k_{\mathrm{cat}}$ of $0.17 \pm 0.04 \mathrm{~s}^{-1}$ and a $\mathrm{K}_{\mathrm{m}}$ of $16 \pm 3 \mu \mathrm{M}$ when using FS-6 as substrate (Table 1). In our hands, the yield of ZnCo-cdMMP7 was about 25\%. The CD spectrum of the heterobimetallic analog showed a similar secondary structural composition as the $\mathrm{ZnZn}$ analog (Figure S2 and Table S1), and the fluorescence spectrum of ZnCo-cdMMP7 
was similar to the as-isolated $\mathrm{Zn}_{2}$-cdMMP7 analog (Figure 3A) suggesting similar tertiary structure for both analogs. A UV-vis difference spectrum of the ZnCo-cdMMP7 analog showed a small, broad peak at $475 \mathrm{~nm}$, which accounts for $\sim 10 \%$ of the cobalt in the sample, which we attribute to $\mathrm{Co}(\mathrm{III})$, and a broad peak at $530 \mathrm{~nm}$, which has an extinction coefficient of $\sim 50 \mathrm{M}^{-}$

${ }^{1} \mathrm{~cm}^{-1}$ (Figure 4)[39, 40, 56, 57]. We assign the latter peak to ligand field transitions of high-spin $\mathrm{Co}(\mathrm{II})$, and the intensity of this peak suggests 6-coordinate $\mathrm{Co}(\mathrm{II})$, suggesting that the $\mathrm{Co}$ (II) binds in the catalytic zinc site. A similar 6-coordinate $\mathrm{Co}(\mathrm{II})$ was previously reported for $\mathrm{Co}$ (II) bound in the catalytic metal binding site of cdMMP16[39].

\subsection{Inhibition studies on cdMMP7 and cdMMP16 with zinc binding group (ZBGs) inhibitors.}

Four ZBGs, acetohydroxamic acid (AHA), maltol, thiomaltol (TM), and allothiomaltol (ATM) (Figure 5), were evaluated as inhibitors of cdMMP7 and cdMMP16. AHA, which is the most common ZBG used in MMPis[10, 30, 32], is a very weak inhibitor of cdMMP7 and cdMMP16, exhibiting $\mathrm{IC}_{50}$ values around $50 \mathrm{mM}$ (Table 4). Maltol was a much better inhibitor, exhibiting $\mathrm{IC}_{50}$ values near $10 \mathrm{mM}$. The replacement of the keto oxygen in maltol with sulfur in thiomaltol resulted in a significant reduction in $\mathrm{IC}_{50}$ values, with cdMMP7 exhibiting an $\mathrm{IC}_{50}$ of $134 \mu \mathrm{M}$ and cdMMP16 exhibiting an $\mathrm{IC}_{50}$ of $30 \mu \mathrm{M}$ (Table 4). Even though $\mathrm{TM}$ is a relatively small inhibitor in size, there is $>4$-fold difference in the $\mathrm{IC}_{50}$ values for cdMMP7 and cdMMP16 (Table 4). Allothiomaltol, which is different from thiomaltol in the fact that the methyl group is trans to the hydroxyl group rather than cis, is a slightly better inhibitor of cdMMP7 and worse inhibitor of cdMMP16. Maltol, thiomaltol, and allothiomaltol are better inhibitors of cdMMP7 and cdMMP16 than the commonly used acetohydroxamic acid. Comparison of our $\mathrm{IC}_{50}$ values with 
those of cdMMP1 (Y. Hao, unpublished) demonstrates selectivity among the four tested ZBGs (Figure 5).

3.5. ITC studies on cdMMP7 and cdMMP16 with thiomaltol and allothiomaltol. To further probe binding of the thiomaltol and allothiomaltol to cdMMP7 and cdMMP16, ITC studies were conducted (Figure 6; Table 5). TM binds to cdMMP7 and cdMMP16 with $\mathrm{K}_{\mathrm{d}}$ values of near 3 $\mu \mathrm{M}$ while affinities for ATM against cdMMP7 and cdMMP16 are 8.5 and $5.8 \mu \mathrm{M}$, respectively. Although the affinities are within the same order of magnitude, thermodynamic parameters determined by ITC highlight a potential difference in binding. The enthalpic contributions to binding for TM against cdMMP7 and cdMMP16 are similar to those for ATM against cdMMP7

and cdMMP16. The range of $K_{d}$ values and negative $\Delta \mathrm{H}$ values near $24 \mathrm{~kJ} \mathrm{~mol}^{-1}$ suggest that although binding of ATM to cdMMP16 is weaker, and binding to cdMMP7 is weaker still, there are fundamental similarities in binding modes for TM/cdMMP7, TM/cdMMP16, ATM/cdMMP7, and ATM/cdMMP16. However, while the TM/cdMMP7, TM/cdMMP16 and ATM/cdMMP16 interactions feature positive $\Delta \mathrm{S}$ values around $25 \mathrm{~J} \mathrm{~mol}^{-1} \mathrm{~K}^{-1}$, the $\Delta \mathrm{S}$ value for ATM against cdMMP7 is smaller and suggests a difference in binding mode compared to TM/cdMMP7, TM/cdMMP16, and ATM/cdMMP16.

\section{Discussion}

MMP7, which is the smallest full-length MMP in terms of size[2, 3], is involved in a number of physiological and pathological processes, due to its involvement in ECM processing and regulation of chemokines and growth factors in various signaling cascades[13, 18]. Since MMP7 lacks a hinge region and a hemopexin-like domain in its C-terminus, the catalytic domain 
(cdMMP7) is the physiologically-relevant analog of the enzyme, once the pro-domain is removed[5]. Therefore, biochemical studies on $\mathrm{Zn}_{2}$ - and metal-substituted analogs of cdMMP7 could shed light on MMP7's role in vivo and provide information needed to design and prepare MMPis in the future.

The NMR solution structures of cdMMP7, MMP7 containing the propeptide, and MMP7/inhibitor complexes have been reported, and all enzymes were prepared using mammalian over-expression cell lines[8]. Two E. coli based over-expression systems were subsequently reported: one system used pET22b[58] and yielded the propeptide, and the other system used pET11a and produced the catalytic domain (cdMMP7)[46]. Both systems resulted in MMP7 being processed into inclusion bodies and required optimized refolding procedures. Compared to cdMMP1[40] and cdMMP16[39], we discovered that the refolding intermediates of cdMMP7 were relatively unstable during dialysis, as the majority of the protein precipitated when using our previous methods. Therefore, we used the method published by Cha to refold cdMMP7, and this method included Brij35 during dialysis steps[46]. Although we successfully obtained active cdMMP7 using this method, the yield of cdMMP7 was low, and cdMMP7 was hydrolyzed (Figure S1A). To address these issues, we modified the Cha method[46] by increasing the Brij35 concentration from $0.05 \%$ to $1 \%$ and by increasing the number of dialysis steps from 1 step to 3 steps with higher concentrations of $\mathrm{Zn}$ (II) (from 20 to $100 \mu \mathrm{M}$ ). Moreover, we found that the starting concentration of inclusion bodies is critical, with any initial concentrations greater than $0.3 \mathrm{mg} / \mathrm{mL}$ resulting in cdMMP7 that exhibited fluorescence spectra that suggested a mixture of folded and unfolded protein and no catalytic activity (Figure S3). We speculate that the higher concentrations of inclusion bodies may have resulted in aggregated forms of the enzyme. 
The kinetic mechanism of cdMMP7 was probed using stopped-flow fluorescence studies. In the first experiment, we monitored intrinsic tryptophan fluorescence emission during hydrolysis of substrate BML-P131. Like earlier studies with cdMMP1 and cdMMP16[39], the fluorescence emission decreased during the initial part of the reaction, and there was an increase in fluorescence emission during the subsequent time period. Fitting of the progress curves to a simple binding kinetic mechanism resulted in microscopic rate constants for cdMMP7 that are much lower than those for MMP1 and MMP16. Steady-state kinetic studies of cdMMP7 with substrate BML-P131 revealed that BML-P131 is a poor substrate for MMP7, most likely due, in part, to the shallow S1' pocket found in MMP7 but not in MMP1 and MMP16[50, 59]. This hypothesis is supported by the fact that the $\mathrm{K}_{\mathrm{s}}$ for BML-P131 binding to cdMMP7 is 10 times larger than that for cdMMP16 (Table 2)[39].

In the second experiment, we monitored the increase in fluorescence emission from product as the substrate FS-6 was hydrolyzed by cdMMP7 and cdMMP16 (Figure $3 \mathrm{C} \& \mathrm{D}$ ). The resulting progress curves were biphasic, with a fast initial "burst" phase followed by a slower phase. The "burst" can be explained by a relatively fast binding of substrate to the enzymes that must result in the rapid increase in fluorescence, presumably due to the loss of quenching between the donor and acceptor in substrate. The much slower hydrolysis of the amide bond in substrate results in the second, slower phase seen in the progress curves in Figure 3. While the kinetic mechanism in Table 3 is the simplest mechanism that models the progress curves, it is likely that there is an ordered released of products, with the product that interacts with the S1' pocket releasing slower. In fact, a kinetic mechanism with ordered product release does in fact simulate the progress curves well, and we believe that the product MCA-L-P-L, which fluoresces, 
interacts with the $\mathrm{S} 1$ ' pocket and releases after the other hydrolysis product, which does not fluoresce.

Co(II)-substituted analogs of MMP1, MMP3, MMP12, and MMP16 have been reported[39, 40, 60, 61]. To prepare heterobimetallic analogs (ZnCo) of MMP, two methods have been used. For MMP1 and MMP16, we dialyzed the as-isolated enzymes against phenanthroline, which chelated $\mathrm{Zn}$ (II) out of the catalytic site but not out of the structural site, and $\mathrm{Co}$ (II) was added to the resulting enzymes[39, 40,60]. Spectroscopic studies were used to confirm that Co(II) was bound in the catalytic site in cdMMP1 and cdMMP16[39, 40]. Cha et al. used this approach to prepare $\mathrm{Co}(\mathrm{II})$-substituted MMP3[60]. Bertini and coworkers prepared a $\mathrm{ZnCo}$ analog of a mutated cdMMP12 by dialyzing the as-isolated enzyme versus millimolar concentrations of $\mathrm{Co}(\mathrm{II})$, and NMR spectra, albeit of the diamagnetic region, were reported[61]. In this work, the ZnCo analog of cdMMP7 was prepared by using the former method, and CD and fluorescence spectra demonstrate that this analog retains the secondary and tertiary structure of the as-isolated ZnZn analog, respectively. UV-Vis spectra revealed broad, weak ligand field transitions, indicating that $\mathrm{Co}(\mathrm{II})$ is 6-coordinate and is bound in the catalytic site. $\mathrm{Co}(\mathrm{II})$ bound in the structural site would have been expected to yield much more intense ligand field transitions[39, 40]. The ZnCo analog is now available to conduct spectroscopic studies on enzyme-inhibitor complexes.

MMPs have been targets for inhibitors for decades[31, 35]. A wide variety of inhibitors have been reported[10, 32], and one common strategy to offer inhibitors is to use a zinc binding group (ZBG) to target the catalytic Zn(II). Substituents have been added to the ZBGs to target substrate binding pockets near the catalytic $\mathrm{Zn}(\mathrm{II})$ in an effort to afford inhibitor selectivity and specificity[30, 33]. The most common ZBG used is AHA, and most MMPs have been tested 
against AHA-based inhibitors[30]. However, most of the MMPs reported to date do not exhibit suitable selectivities or specificities to be clinically useful. In addition, our studies herein and the studies of others $[62,63]$ demonstrate that AHA is a very weak binding inhibitor of the MMPs, warranting efforts to test other ZBGs. Indeed, our data presented herein demonstrate that maltol, thiomaltol, and allothiomaltol are all better inhibitors of cdMMP7, cdMMP16, and cdMMP1 (Table 4 and Figure 5). ITC measurements demonstrate that thiomaltol and allothiomaltol bind to cdMMP7 and cdMMP16 with low micromolar $\mathrm{K}_{\mathrm{d}}$ values (Table 5). Despite similar structures, there does appear to be, albeit modest, selectivity of maltol, thiomaltol, and allothiomaltol binding to cdMMP7 and cdMMP16 (Figure 5), suggesting that these compounds could serve as better ZBGs, when compared to AHA, for future MMP inhibitors. The trends observed in the ITC data suggest a commonality between TM binding to cdMMP7 and cdMMP16. Docking studies using RosettaDock found that TM binding to both cdMMP7 and cdMMP16 feature thione sulfur binding to the catalytic zinc, with possible bidentate binding to zinc via the TM hydroxyl oxygen and burying of the TM methyl group into the S1' pocket (Figure $6 \mathrm{E} \& \mathrm{~F}$ ). In contrast, the ITC data suggests a weaker, yet similar affinity for ATM against cdMMP16 and again weaker affinity of ATM against cdMMP7. The docking studies suggest that decreased affinity of ATM against cdMMP16 may be due to monodentate zinc binding through the ATM thione sulfur paired with burying of the ATM methyl group into the S1' pocket of cdMMP16 (Figure 6F). In contrast, the docking studies indicate that the further decrease in affinity observed by ITC for ATM against cdMMP7 may be due to a binding orientation that precludes burying of the ATM methyl group into the cdMMP7 S1' pocket, despite a possible bidentate zinc binding mode via the ATM thione sulfur and hydroxyl oxygen. Differences in binding mode for ATM/cdMMP7, in comparison to TM/cdMMP7, TM/cdMMP16, and ATM/cdMMP16 is 
consistent with a different entropic contribution to binding for ATM/cdMMP7 despite similarities in enthalpic contributions across the four ZBGs. In combination, the ITC data and docking studies indicate that inhibitor binding to MMPs is dictated both by inhibitor structure and the local protein structure surrounding the $\mathrm{S} 1$ ' pocket. Future structural, biophysical, and spectroscopic studies of ZBG/cdMMP7 and ZBG/cdMMP16 complexes will be an important step to capitalizing upon these different binding modes to provide a potential avenue for introducing additional selectivity and specificity against different MMPs.

With the preparation of a heterobimetallic analog of cdMMP7, we can now probe inhibitor binding using future spectroscopic studies, rather than relying completely on crystal structures of enzyme-inhibitor complexes. We hypothesize that the successful preparation of suitably specific and selective MMPis will require iterative design of inhibitors using tandem kinetic and structural studies on one or more MMPs from each of the distinct MMP subclasses. The generation of a stable heterobimetallic $\mathrm{ZnCo}$ analog of cdMMP7 will yield less complicated spectroscopic results and remove the need to subtract spectroscopic signals associated with the structural metal binding site. With the preparation and characterization of a stable, wellcharacterized analog of MMP7 (and our previous work on MMP1[40], MMP16[39], and MMP3 (unpublished)), we need only a MMP from the two remaining classes of MMPs (gelatinases and elastases) to possess a MMP from each of representative MMP classes. Efforts to prepare and characterize cdMMP9 and cdMMP12 are underway. 


\section{Abbreviations}

AHA, acetohydroxamic acid

ATM, allothiomaltol

$\mathrm{CD}$, circular dichroism

cdMMP, catalytic domain of matrix metalloproteinase

DNP, 2,4-dinitrophenyl

ECM, extracellular matrix

EDTA, ethylenediaminetetraacetic acid

Hepes, 4-(2-hydroxyethyl)-1-piperazineethanesulfonic acid

IPTG, isopropyl- $\beta$ - $D$-thiogalactoside

ITC, isothermal titration calorimetry

Kan, kanamycin

LB, Luria-Bertani

MALDI-TOF, matrix assisted laser desorption ionization - time of flight

MCA, (7-methoxycoumarin-4-yl) acetic acid

MMP, matrix metalloproteinase

MMPis, MMP inhibitors

MSS, musculoskeletal syndrome

MT-MMP, membrane-type MMP

OP, phenanthroline

SAR, structure activity relationship

SDS, sodium dodecyl sulfate

TCEP, tris(2-carboxyethyl)phosphine 
Tris, tris(hydroxymethyl)aminomethane

TM, thiomaltol

ZBG, zinc binding group

\section{Acknowledgements}

We thank Dr. Carole L. Wilson (University of Washington) for providing a pGEM7-based plasmid containing the full-length MMP7 sequence. We thank Dr. Seth M. Cohen (University of California San Diego) for providing the ZBGs. We thank Dr. Neil D. Danielson (Miami University) for assistance with fluorescence spectroscopic experiments. ITC, ICP-AES, and CD spectroscopic studies were supported by facilities in the Department of Chemistry and Biochemistry at Miami University.

\section{Reference}

[1] C. Tallant, A. Marrero, F.X. Gomis-Ruth, Biochim. Biophys. Acta 1803 (2010) pp. 20-28.

[2] H. Nagase, R. Visse, G. Murphy, Cardiovasc. Res., 69 (2006) pp. 562-573.

[3] W.H. Rodgers, L.M. Matrisian, M. Navre, L.C. Giudice, F. Gorstein, Am. J. Obstet. Gynecol., 168 (1993) pp. 253-260.

[4] S.E. Dunsmore, U.K. Saarialho-Kere, J.D. Roby, C.L. Wilson, L.M. Matrisian, H.G. Welgus, W.C. Parks, J. Clin. Invest., 102 (1998) pp. 1321.

[5] T. Crabbe, F. Willenbrock, D. Eaton, P. Hynds, A.F. Carne, G. Murphy, A.J. Docherty, Biochemistry, 31 (1992) pp. 8500-8507.

[6] H. Nagase, J.J. Enghild, K. Suzuki, G. Salvesen, Biochemistry, 29 (1990) pp. 5783-5789. 
[7] A. Park, L. Matrisian, A. Kells, R. Pearson, Z. Yuan, M. Navre, J. Biol. Chem., 266 (1991) pp. 1584-1590.

[8] M.F. Browner, W.W. Smith, A.L. Castelhano, Biochemistry, 34 (1995) pp. 6602-6610.

[9] G.E. Terp, G. Cruciani, I.T. Christensen, F.S. Jørgensen, J. Med. Chem., 45 (2002) pp. 26752684.

[10] J.A. Jacobsen, J.L.M. Jourden, M.T. Miller, S.M. Cohen, Biochim. Biophys. Acta-Mol. Cell. Res. 1803 (2010) pp. 72-94.

[11] C. Tallant, R. García-Castellanos, U. Baumann, F.X. Gomis-Rüth, J. Biol. Chem., 285 (2010) pp. 13951-13957.

[12] C.K. Engel, B. Pirard, S. Schimanski, R. Kirsch, J. Habermann, O. Klingler, V. Schlotte, K.U. Weithmann, K.U. Wendt, Chem. Biol., 12 (2005) pp. 181-189.

[13] M. Ii, H. Yamamoto, Y. Adachi, Y. Maruyama, Y. Shinomura, Exp. Biol. Med., 231 (2006) pp. 20-27.

[14] C.L. Wilson, L.M. Matrisian, Int. J. Biochem. Cell Biol., 28 (1996) pp. 123-136.

[15] J.D. Mott, Z. Werb, Curr. Opin. Cell Biol., 16 (2004) pp. 558-564.

[16] A. Agrawal, D. Romero-Perez, J.A. Jacobsen, F.J. Villarreal, S.M. Cohen, ChemMedChem, 3 (2008) pp. 812-820.

[17] G.S. Butler, C.M. Overall, Periodontol. 2000, 63 (2013) pp. 123-148.

[18] A. Dufour, C.M. Overall, Trends Pharmacol. Sci., 34 (2013) pp. 233-242.

[19] N. Fortelny, S. Yang, P. Pavlidis, P.F. Lange, C.M. Overall, Nucleic Acids Res., 43 (2015) pp. D290-D297.

[20] Y. Yokoyama, F. Grünebach, S.M. Schmidt, A. Heine, M. Häntschel, S. Stevanovic, H.-G. Rammensee, P. Brossart, Clin. Cancer. Res., 14 (2008) pp. 5503-5511. 
[21] A. Szklarczyk, G. Oyler, R. McKay, C. Gerfen, K. Conant, J. Neurochem., 102 (2007) pp. $1256-1263$.

[22] S.H. Prior, Y.G. Fulcher, R.K. Koppisetti, A. Jurkevich, S.R. Van Doren, Structure, 23 (2015) pp. 2099-2110.

[23] S. Chaturvedi, R. Hass, Mech. Ageing Dev., 132 (2011) pp. 213-219.

[24] C.L. Wilson, K.J. Heppner, P.A. Labosky, B.L. Hogan, L.M. Matrisian, Proc. Natl. Acad. Sci. U.S.A. 94 (1997) pp. 1402-1407.

[25] A. Fukuda, S.C. Wang, J.P. Morris, A.E. Folias, A. Liou, G.E. Kim, S. Akira, K.M. Boucher, M.A. Firpo, S.J. Mulvihill, Cancer Cell, 19 (2011) pp. 441-455.

[26] Y.T. Konttinen, M. Ainola, H. Valleala, J. Ma, H. Ida, J. Mandelin, R.W. Kinne, S.

Santavirta, T. Sorsa, C. López-Otín, Ann. Rheum. Dis., 58 (1999) pp. 691-697.

[27] U. Saarialho-Kere, Arch. Dermatol. Res.. 290 (1998) pp. S47-S54.

[28] U.K. Saarialho-Kere, M. Vaalamo, P. Puolakkainen, K. Airola, W.C. Parks, M.-L.

Karjalainen-Lindsberg, Am. J. Pathol, 148 (1996) pp. 519.

[29] B. Wielockx, C. Libert, C. Wilson, Cytokine Growth Factor Rev., 15 (2004) pp. 111-115.

[30] R.E. Vandenbroucke, C. Libert, Nat. Rev. Drug. Discov., 13 (2014) pp. 904-927.

[31] B. Fingleton, Proc. Semin. Cell Dev. Biol. (2008) Elsevier 19 61-68.

[32] J. Cathcart, A. Pulkoski-Gross, J. Cao, Genes Dis., 2 (2015) pp. 26-34.

[33] B. Fabre, A. Ramos, B. de Pascual-Teresa, J. Med. Chem., vol. 57, 2014, pp. 10205-10219.

[34] L. Aureli, M. Gioia, I. Cerbara, S. Monaco, G.F. Fasciglione, S. Marini, P. Ascenzi, A.

Topai, M. Coletta, Curr. Med. Chem., 15 (2008) pp. 2192-2222.

[35] J.W. Skiles, N.C. Gonnella, A.Y. Jeng, Curr. Med. Chem., 11 (2004) pp. 2911-2977. 
[36] S. Rowsell, P. Hawtin, C.A. Minshull, H. Jepson, S.M. Brockbank, D.G. Barratt, A.M.

Slater, W.L. McPheat, D. Waterson, A.M. Henney, J. Mol. Biol., 319 (2002) pp. 173-181.

[37] B.S. Antharavally, K.A. Mallia, P. Rangaraj, P. Haney, P.A. Bell, Anal. Biochem., 385 (2009) pp. 342-345.

[38] Gasteiger, E., Hoogland, C., Gattiker, A., Duvaud, S. e., Wilkins, M. R., Appel, R. D. and Bairoch, A. (2005) Protein identification and analysis tools on the ExPASy server. Springer.

[39] F. Meng, H. Yang, M. Aitha, S. George, D.L. Tierney, M.W. Crowder, J. Biol. Inorg. Chem., 156 (2016) pp. 1-13.

[40] H. Yang, K. Makaroff, N. Paz, M. Aitha, M.W. Crowder, D.L. Tierney, Biochemistry, 54 (2015) pp. 3631-3639.

[41] L. Michaelis, M.L. Menten, Biochem., 49 (1913) pp. 352.

[42] H. Motulsky, GraphPad Software (2007) pp. 1-26.

[43] J. Galvez, R. Varon, F.G. Canovas, F.G. Carmona, J. Theor. Biol., 94 (1982) pp. 413-420.

[44] P. Kuzmič, Methods Enzymol., 467 (2009) pp. 247-280.

[45] E.L. King, C. Altman, J. Phys. Chem., 60 (1956) pp. 1375-1378.

[46] J. Cha, M.V. Pedersen, D.S. Auld, Biochemistry, 35 (1996) pp. 15831-15838.

[47] L. Whitmore, B. Wallace, Nucleic Acids Res., 32 (2004) pp. W668-W673.

[48] D.P. Martin, P.G. Blachly, A.R. Marts, T.M. Woodruff, C.S.A. de Oliveira, J.A.

McCammon, D.L. Tierney, S.M. Cohen, J. Am. Chem. Soc., 136 (2014) pp. 5400-5406.

[49] Milev, S., Bosshard, H. R., and Jelesarov, I., Biochemistry, 44 (2005) pp. 285-293.

[50] R. Lang, M. Braun, N.E. Sounni, A. Noël, F. Frankenne, J.-M. Foidart, W. Bode, K. Maskos, J. Mol. Biol., 336 (2004) pp. 213-225. 
[51] S. Chaudhury, M. Berrondo, B.D. Weitzner, P. Muthu, H. Bergman, J.J. Gray, PLoS One, 6 (2011) pp. e22477.

[52] P.C. Hawkins, A.G. Skillman, G.L. Warren, B.A. Ellingson, M.T. Stahl, J. Chem. Inf. Model., 50 (2010) pp. 572-584.

[53] I.W. Davis, D. Baker, J. Mol. Biol., 385 (2009) pp. 381-392.

[54] J. Meiler, D. Baker, Proteins: Struct., Funct., Bioinf., 65 (2006) pp. 538-548.

[55] H. Oneda, K. Inouye, J. Biochem., 126 (1999) pp. 905-911.

[56] R.M. Breece, A. Costello, B. Bennett, T.K. Sigdel, M.L. Matthews, D.L. Tierney, M.W. Crowder, J. Biol. Chem., 280 (2005) pp. 11074-11081.

[57] I. Bertini, C. Luchinat, Acc. Chem. Res., 16 (1983) pp. 272-279.

[58] Y. Muta, N. Yasui, Y. Matsumiya, M. Kubo, K. Inouye, Biosci. Biotechnol. Biochem., 74 (2010) pp. 2515-2517.

[59] B. Lovejoy, A.R. Welch, S. Carr, C. Luong, C. Broka, R.T. Hendricks, J.A. Campbell, K.A. Walker, R. Martin, H. Van Wart, Nat. Struct. Mol. Biol., 6 (1999) pp. 217-221.

[60] J. Cha, M.V. Sørensen, Q.-Z. Ye, D.S. Auld, J. Biol. Inorg. Chem., 3 (1998) pp. 353-359.

[61] I. Bertini, M. Fragai, Y.M. Lee, C. Luchinat, B. Terni, Angew. Chem. Int. Ed., 43 (2004) pp. 2254-2256.

[62] D.T. Puerta, J.A. Lewis, S.M. Cohen, J. Am. Chem. Soc., 126 (2004) pp. 8388-8389.

[63] D.T. Puerta, M.O. Griffin, J.A. Lewis, D. Romero-Perez, R. Garcia, F.J. Villarreal, S.M.

Cohen, J. Biol. Inorg. Chem,. 11 (2006) pp. 131-138. 
Tables

Table 1: Steady-state kinetic constants and metal content for recombinant cdMMP7 analogs

\begin{tabular}{lccccc}
\hline & $\boldsymbol{k}_{c \text { at }}\left(\mathbf{s}^{-\mathbf{1}}\right)$ & $\mathbf{K}_{\mathbf{m}}(\boldsymbol{\mu M})$ & $\boldsymbol{k}_{\text {cat }} / \mathbf{K}_{\mathbf{M}}\left(\mathbf{s}^{-1} \boldsymbol{\mu} \mathbf{M}^{-\mathbf{1}}\right)$ & \multicolumn{2}{c}{ Metal/protein ratio } \\
\cline { 5 - 6 } & & & & $\mathbf{Z n}$ & $\mathbf{C o}$ \\
\hline $\begin{array}{l}\mathbf{Z n} \text {-cdMMP7 } \\
\text { (as-isolated) }\end{array}$ & $0.40 \pm 0.02$ & $23 \pm 2$ & 0.017 & $1.8 \pm 0.1$ & $<0.1$ \\
\hline $\mathbf{Z n C o - c d M M P 7 ~}$ & $0.17 \pm 0.04$ & $16 \pm 3$ & 0.011 & 0.7 & 1.2 \\
\hline
\end{tabular}

Steady-state kinetic reactions were conducted in $50 \mathrm{mM}$ Hepes, $\mathrm{pH} 7.5$, containing $100 \mathrm{mM}$

$\mathrm{NaCl}$ and $5 \mathrm{mM} \mathrm{CaCl} 2$ at $25{ }^{\circ} \mathrm{C}$. The substrate was MCA-fluorescence peptide (Sigma Aldrich

FS-6), and the concentration of $\mathrm{Zn}_{2}$-cdMMP7 was $10 \mu \mathrm{M}$, and the concentration of $\mathrm{ZnCo-}$ cdMMP7 was $20 \mu \mathrm{M}$ 
Table 2. Microscopic kinetic constants of the hydrolysis of BML-P131 by $\mathrm{Zn}_{2}$-cdMMP7

\begin{tabular}{|c|c|c|c|c|}
\hline & & $\underset{k_{-1}}{\stackrel{k_{1}}{\rightleftharpoons}}$ & $\stackrel{k_{2}}{\longrightarrow}$ & \\
\hline & $k_{1}\left(\mu \mathrm{M}^{-1} \mathrm{~s}^{-1}\right)$ & $k_{-1}\left(\mathrm{~s}^{-1}\right)$ & $k_{2}\left(\mathrm{~s}^{-1}\right)$ & $\mathbf{K}_{\mathrm{s}}(\mu \mathbf{M})$ \\
\hline cdMMP7 & 0.0079 & 0.83 & 0.001 & 30.5 \\
\hline cdMMP1 & 0.35 & 10.7 & 2.0 & NR \\
\hline cdMMP16 & 0.30 & $1 \times 10^{-6}$ & 2.7 & 3.71 \\
\hline
\end{tabular}

Stopped-flow fluorescence reactions were conducted in $50 \mathrm{mM}$ Hepes, $\mathrm{pH} 7.5$, containing 100 $\mathrm{mM} \mathrm{NaCl}$ and $5 \mathrm{mM} \mathrm{CaCl}_{2}$ at $25{ }^{\circ} \mathrm{C}$. Progress curves for data in Figure 3B were generated by using Dynafit 3.4[44]. Data on cdMMP1 and cdMMP16 were previously published.[39, 40] NR - not reported. 
Table 3. Microscopic kinetic constants of as-isolated $\mathrm{Zn}_{2}$-cdMMP7 and $\mathrm{Zn}_{2}$-cdMMP16 using FS-6 as substrate from substrate-emission stopped-flow studies

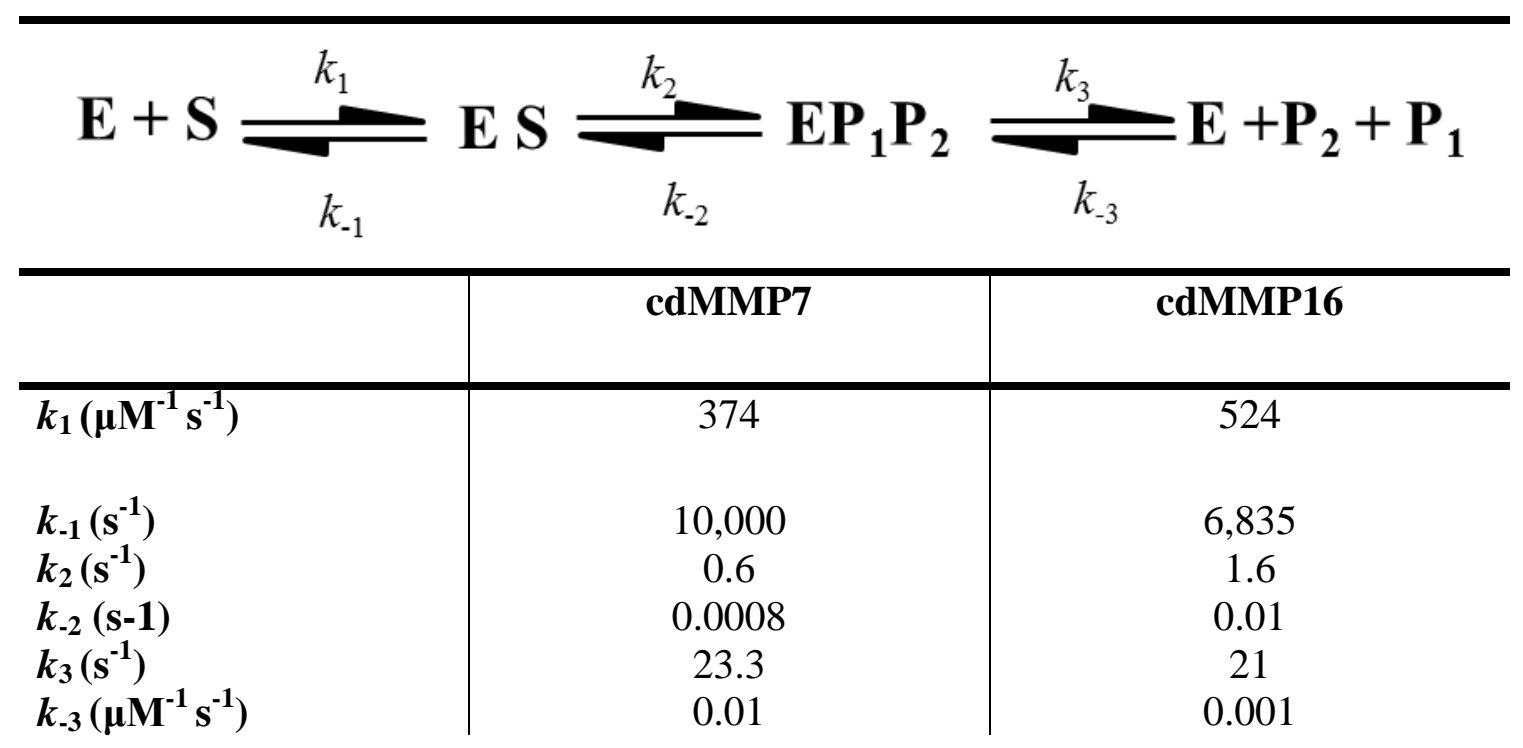

Reactions were conducted in $50 \mathrm{mM}$ Hepes, pH 7.5, containing $100 \mathrm{mM} \mathrm{NaCl}$ and $5 \mathrm{mM} \mathrm{CaCl}_{2}$ at $25{ }^{\circ} \mathrm{C}$. Progress curves for data in Figure 3C and Figure 3D were produced by using Dynafit 3.4 [44]. 
Table 4. $\mathrm{IC}_{50}$ values of AHA, maltol, TM, and ATM as inhibitors of cdMMP16 and cdMMP7.

\begin{tabular}{|c|c|c|c|c|}
\hline & \multicolumn{2}{|c|}{ cdMMP7 } & \multicolumn{2}{|c|}{ cdMMP16 } \\
\hline & $\mathrm{IC}_{50}(\mu \mathrm{M})$ & Potency vs AHA & $\mathrm{IC}_{50}(\mu \mathrm{M})$ & Potency vs AHA \\
\hline AHA & $55,800 \pm 1,730$ & ------ & $41,000 \pm 1,000$ & ----- \\
\hline Maltol & $7,308 \pm 890$ & 7.6 & $10,300 \pm 1,900$ & 4 \\
\hline TM & $134 \pm 13$ & 416 & $30 \pm 5$ & 1370 \\
\hline ATM & $103 \pm 9$ & 544 & $43 \pm 3$ & 953 \\
\hline
\end{tabular}

Inhibition studies were conducted with $10 \mu \mathrm{M}$ enzyme and $10 \mu \mathrm{M} \mathrm{MCA}$ substrate at $25^{\circ} \mathrm{C}$ in 50 mM Hepes, $\mathrm{pH}$ 7.5, containing $100 \mathrm{mM} \mathrm{NaCl}$ and $5 \mathrm{mM} \mathrm{CaCl}_{2}$. 
Table 5. ITC measurements on cdMMP7 and cdMMP16 using ATM and TM as binding groups.

\begin{tabular}{lllllll}
\hline MMPs & ZBGs & $\begin{array}{l}\mathbf{K}_{\mathbf{d}} \\
(\boldsymbol{\mu M})\end{array}$ & $\begin{array}{l}\boldsymbol{\Delta} \mathbf{H} \\
(\mathbf{k J} / \mathbf{m o l})\end{array}$ & $\begin{array}{l}\Delta \mathbf{S} \\
(\mathbf{J} / \mathbf{m o l K})\end{array}$ & $\begin{array}{l}\Delta \mathbf{G} \\
(\mathbf{k J} / \mathbf{m o l})\end{array}$ & $\mathbf{n}$ \\
\hline $\mathbf{Z n} \mathbf{n}_{2}$-MMP16 & TM & 3.0 & -22 & 33 & -32 & 1.0 \\
& ATM & 5.8 & -24 & 21 & -30 & 0.9 \\
\hline Znn-MMP7 & TM & 3.2 & -23 & 25 & -30 & 1.1 \\
& ATM & 8.5 & -26 & 11 & -29 & 1.0
\end{tabular}

The buffer used in these experiments was $50 \mu \mathrm{M}$ using $50 \mathrm{mM}$ Hepes, $\mathrm{pH} 7.5$, containing 100

$\mathrm{mM} \mathrm{NaCl}$ and $5 \mathrm{mM} \mathrm{CaCl}_{2}$ for cdMMP16 and $50 \mathrm{mM}$ Tris, $\mathrm{pH} 7.5$, containing $5 \mathrm{mM} \mathrm{ZnCl}_{2}$, and $0.1 \%(w / v)$ Brij35 for cdMMP7. The enzyme concentration for MMP16 and MMP7 was $50 \mu \mathrm{M}$. 


\section{Figure Captions}

Figure 1: Crystal structure of $\mathrm{Zn}_{2}$-cdMMP7 (PDB ID 1MMB) [8]. $\mathrm{Zn}(\mathrm{II})$ and $\mathrm{Ca}$ are colored gray and blue, respectively. Right: $\mathrm{Zn}$ (II) binding sites are shown with metal binding amino acids labeled and S1' substrate binding pockets shown in magenta color [12]. The Met-turn is shown in orange [8].

Figure 2: Structure of substrate A. FS-6 substrate and B. BML-P131 substrate. The scissile bonds are labeled with the red-dashed box.

Figure 3: (A): Fluorescence emission spectra of $\mathrm{ZnCo-cdMMP7} \mathrm{(dash)} \mathrm{and} \mathrm{as-isolated} \mathrm{Zn}_{2^{-}}$ cdMMP7 (solid).(B): Stopped-flow fluorescence traces of reactions of $\mathrm{Zn}_{2}$-cdMMP7 and with substrate BML-P131. The buffer was $50 \mathrm{mM}$ Hepes, $\mathrm{pH} 7.5$, containing $100 \mathrm{mM} \mathrm{NaCl}$ and 5 $\mathrm{mM} \mathrm{CaCl} 2$, and the reactions were conducted at $25{ }^{\circ} \mathrm{C}$. The simulated progress curves, which were generated by using Dynafit 3.4 and the kinetic mechanism in Table 2, are represented by the solid lines, and the stopped-flowed fluorescence data are represented by the symbols. The final concentration of cdMMP7 in these reactions was $10 \mu \mathrm{M}$. (C) and (D): Stopped-flow fluorescence traces of reactions of $\mathrm{A} \mathrm{Zn}_{2}$-cdMMP7 and B $\mathrm{Zn}_{2}$-cdMMP16 with FS-6 fluorescent substrate. The buffer was $50 \mathrm{mM}$ Hepes, $\mathrm{pH} 7.5$, containing $100 \mathrm{mM} \mathrm{NaCl}$ and $5 \mathrm{mM} \mathrm{CaCl}$, and the reactions were conducted at $25{ }^{\circ} \mathrm{C}$. The simulated progress curves are represented by the solid lines, and the stopped-flow fluorescence data are represented by the symbols. The final concentrations of the cdMMPs were $0.5 \mu \mathrm{M}$.

Figure 4: Uv-vis spectrum of of the ZnCo-cdMMP7 analog( right up corner) and UV-Vis difference (spectrum of $\mathrm{ZnCo}$ analog minus the spectrum of metal-free enzyme) spectrum in 50 mM Hepes, $\mathrm{pH} 7.5$, containing $100 \mathrm{mM} \mathrm{NaCl}$ and $5 \mathrm{mM} \mathrm{CaCl}$. 
Figure 5: (Left),Structures of zinc binding group (ZBG) inhibitors used in this study (from left to right): acetohydroxamic acid (AHA), maltol, thiomaltol (TM), and allothiomaltol (ATM). (Right), Inhibitory potency of AHA, maltol, TM, and ATM against cdMMP7 and cdMMP16 relative to cdMMP1 (H. Yang, unpublished data).

Figure 6: ITC results of ZBG binding to cdMMP7 and cdMMP16. Titration of TM (A) and ATM (B) into $50 \mu \mathrm{M} \mathrm{Zn}_{2}$-cdMMP16 in $50 \mathrm{mM}$ Hepes, $\mathrm{pH}$ 7.5, containing $100 \mathrm{mM} \mathrm{NaCl}$ and 5 $\mathrm{mM} \mathrm{CaCl} l_{2}$ at $25{ }^{\circ} \mathrm{C}$. Titrations of $\mathrm{TM}(\mathbf{C}) / \mathrm{ATM}(\mathbf{D})$ into $50 \mu \mathrm{M} \mathrm{Zn} \mathrm{Zn}_{2}$-cdMMP7 in $50 \mathrm{mM}$ Tris, pH 7.5, containing $5 \mathrm{mM} \mathrm{CaCl}_{2}$ and $0.1 \%$ Brij 35 at $25{ }^{\circ} \mathrm{C}$. Representative binding modes from docking simulations (E) and (F). RosettaDock simulations of cdMMP7 (E) with TM (cyan) and ATM (blue). RosettaDock simulations of cdMMP16 (F) with TM (purple) and ATM (pink). Throughout, protein backbones are show as cartoons, relevant interacting side chains shown as lines, bound ions shown as spheres, and TM and ATM are shown as sticks. 


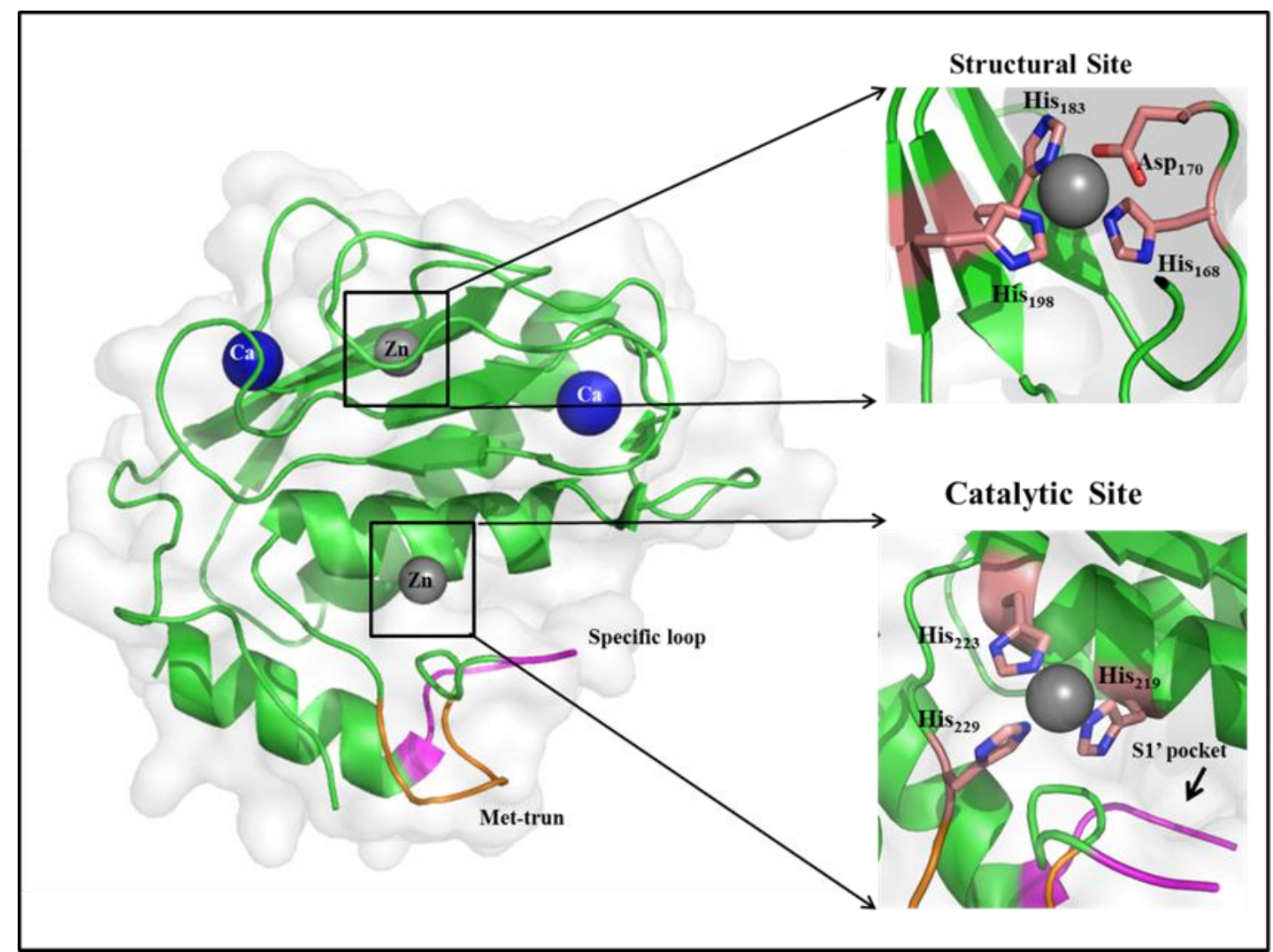

Figure 1: Crystal structure of $\mathrm{Zn}_{2}$-cdMMP7 (PDB \#1MMB) [8]. $\mathrm{Zn}(\mathrm{II})$ and $\mathrm{Ca}$ are colored gray and blue, respectively. Right: $\mathrm{Zn}$ (II) binding sites are shown with metal binding amino acids labeled and S1' substrate binding pockets shown in magenta color [12]. The Met-turn is shown in orange[8]. 
A

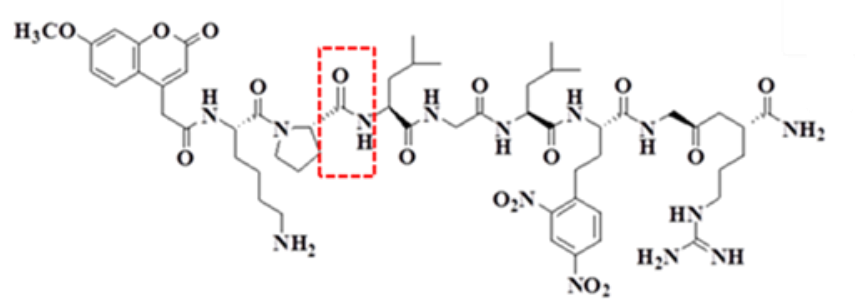

B

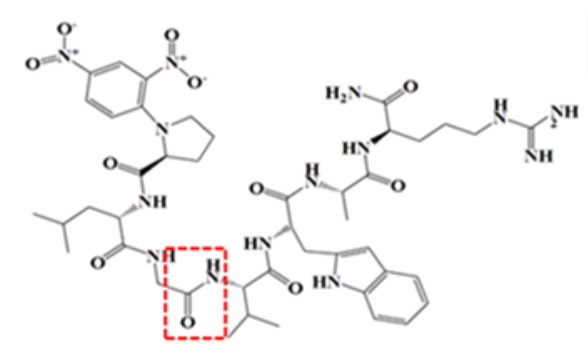

Figure 2: Structure of substrate A. FS-6 substrate and B. BML-P131 substrate. The scissile bonds are labeled with the red-dashed box. 

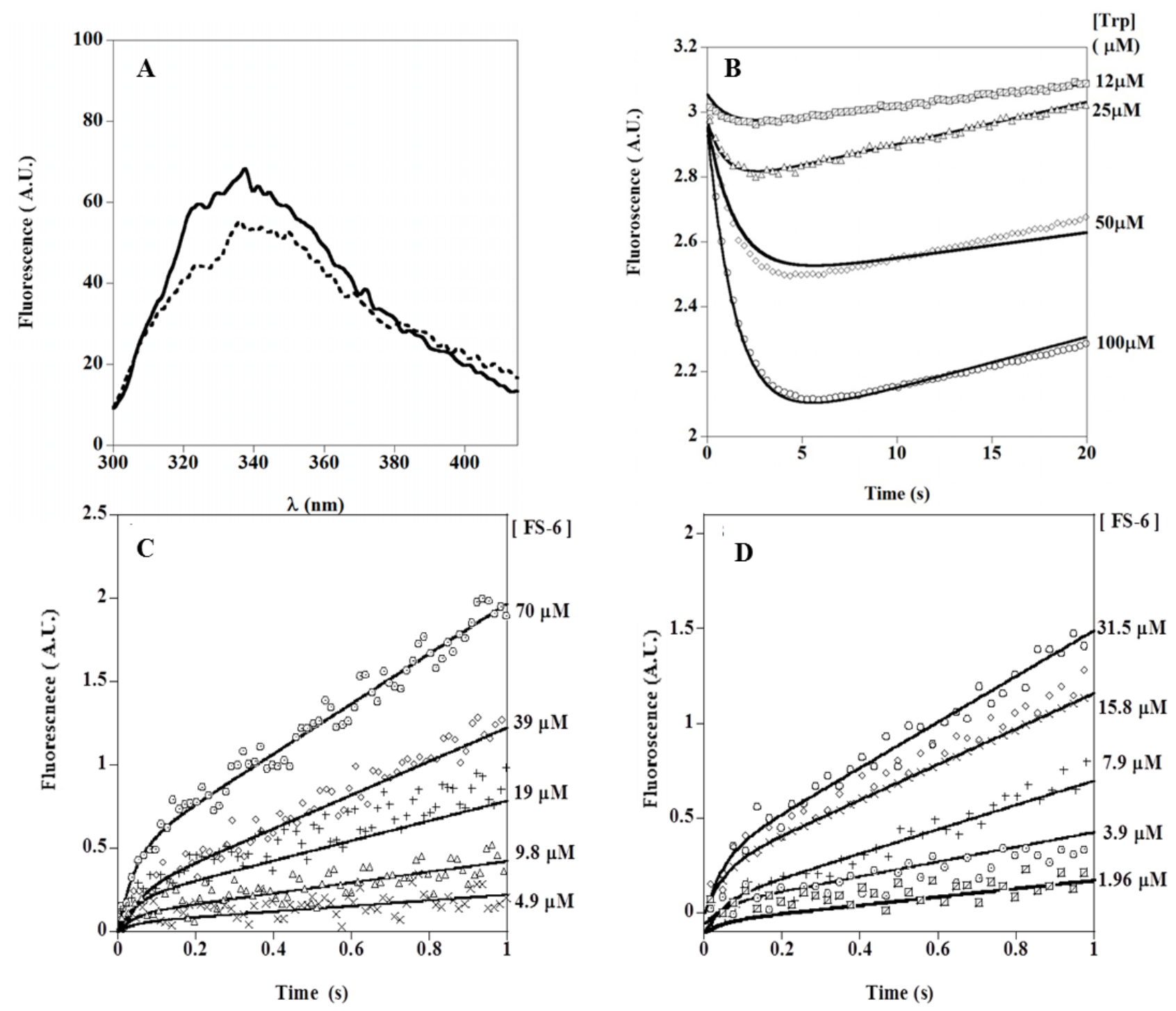

Figure 3: (A): Fluorescence emission spectra of $\mathrm{ZnCo-cdMMP7} \mathrm{(dash)} \mathrm{and} \mathrm{as-isolated} \mathrm{Zn}_{2^{-}}$ cdMMP7 (solid).(B): Stopped-flow fluorescence traces of reactions of $\mathrm{Zn}_{2}$-cdMMP7 and with substrate BML-P131. The buffer was $50 \mathrm{mM}$ Hepes, $\mathrm{pH} 7.5$, containing $100 \mathrm{mM} \mathrm{NaCl}$ and 5 $\mathrm{mM} \mathrm{CaCl}{ }_{2}$, and the reactions were conducted at $25{ }^{\circ} \mathrm{C}$. The simulated progress curves, which were generated by using Dynafit 3.4 and the kinetic mechanism in Table 2, are represented by the solid lines, and the stopped-flowed fluorescence data are represented by the symbols. The final concentration of cdMMP7 in these reactions was $10 \mu \mathrm{M}$. (C) and (D): Stopped-flow 
fluorescence traces of reactions of A Zn2-cdMMP7 and B Zn2-cdMMP16 with FS-6 fluorescent substrate. The buffer was $50 \mathrm{mM}$ Hepes, $\mathrm{pH} 7.5$, containing $100 \mathrm{mM} \mathrm{NaCl}$ and $5 \mathrm{mM} \mathrm{CaCl} 2$, and the reactions were conducted at $25 \mathrm{oC}$. The simulated progress curves are represented by the solid lines, and the stopped-flow fluorescence data are represented by the symbols. The final concentrations of the cdMMPs were $0.5 \mu \mathrm{M}$. 


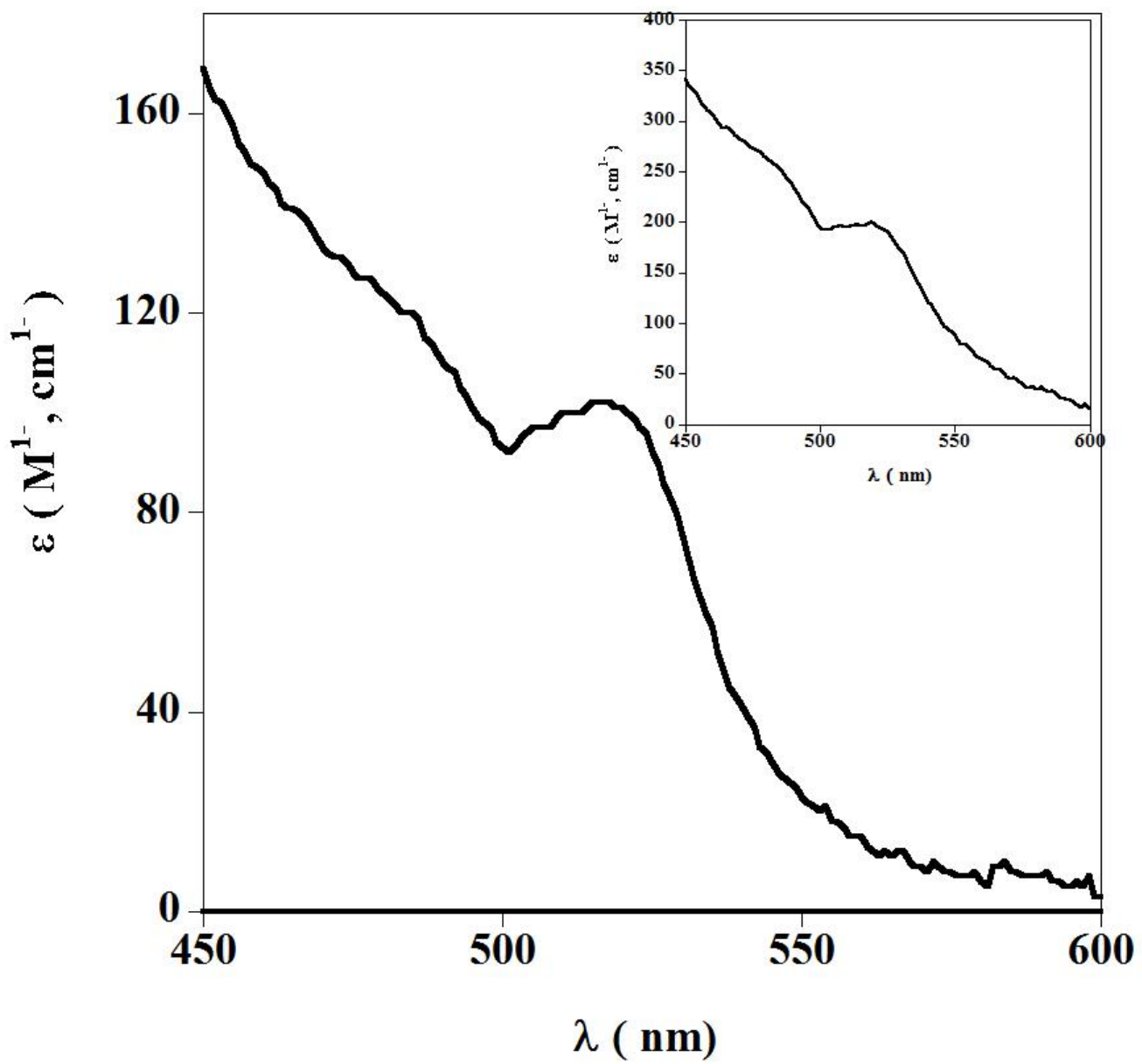

Figure 4: Uv-vis spectrum of of the ZnCo-cdMMP7 analog( right top corner) and UV-Vis difference (spectrum of $\mathrm{ZnCo}$ analog minus the spectrum of metal-free enzyme) spectrum in 50 $\mathrm{mM}$ Hepes, $\mathrm{pH} 7.5$, containing $100 \mathrm{mM} \mathrm{NaCl}$ and $5 \mathrm{mM} \mathrm{CaCl}_{2}$. 


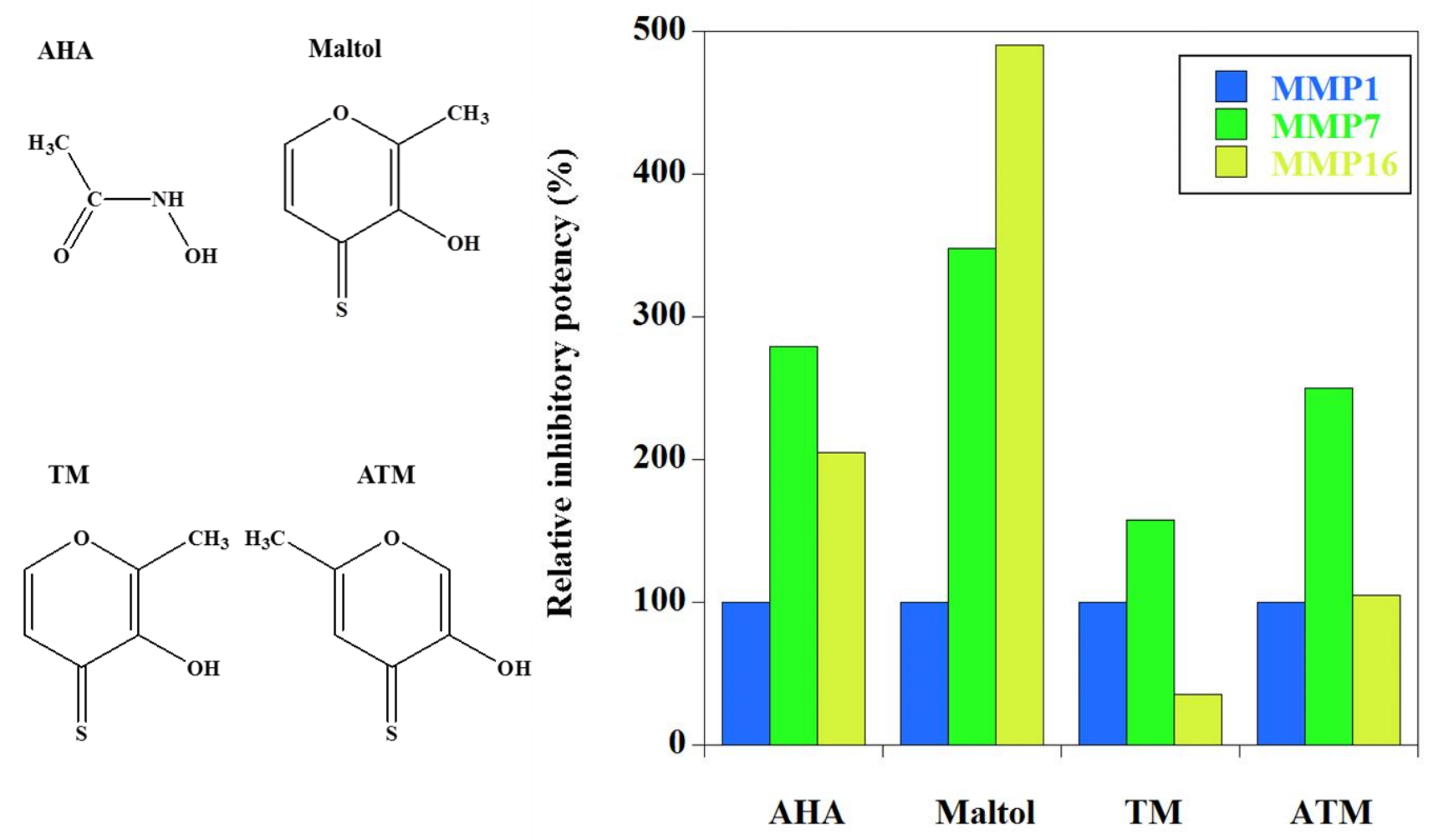

Figure 5: (Left),Structures of zinc binding group (ZBG) inhibitors used in this study (from left to right): acetohydroxamic acid (AHA), maltol, thiomaltol (TM), and allothiomaltol (ATM). (Right), Inhibitory potency of AHA, maltol, TM, and ATM against cdMMP7 and cdMMP16 relative to cdMMP1 (H. Yang, unpublished data). 

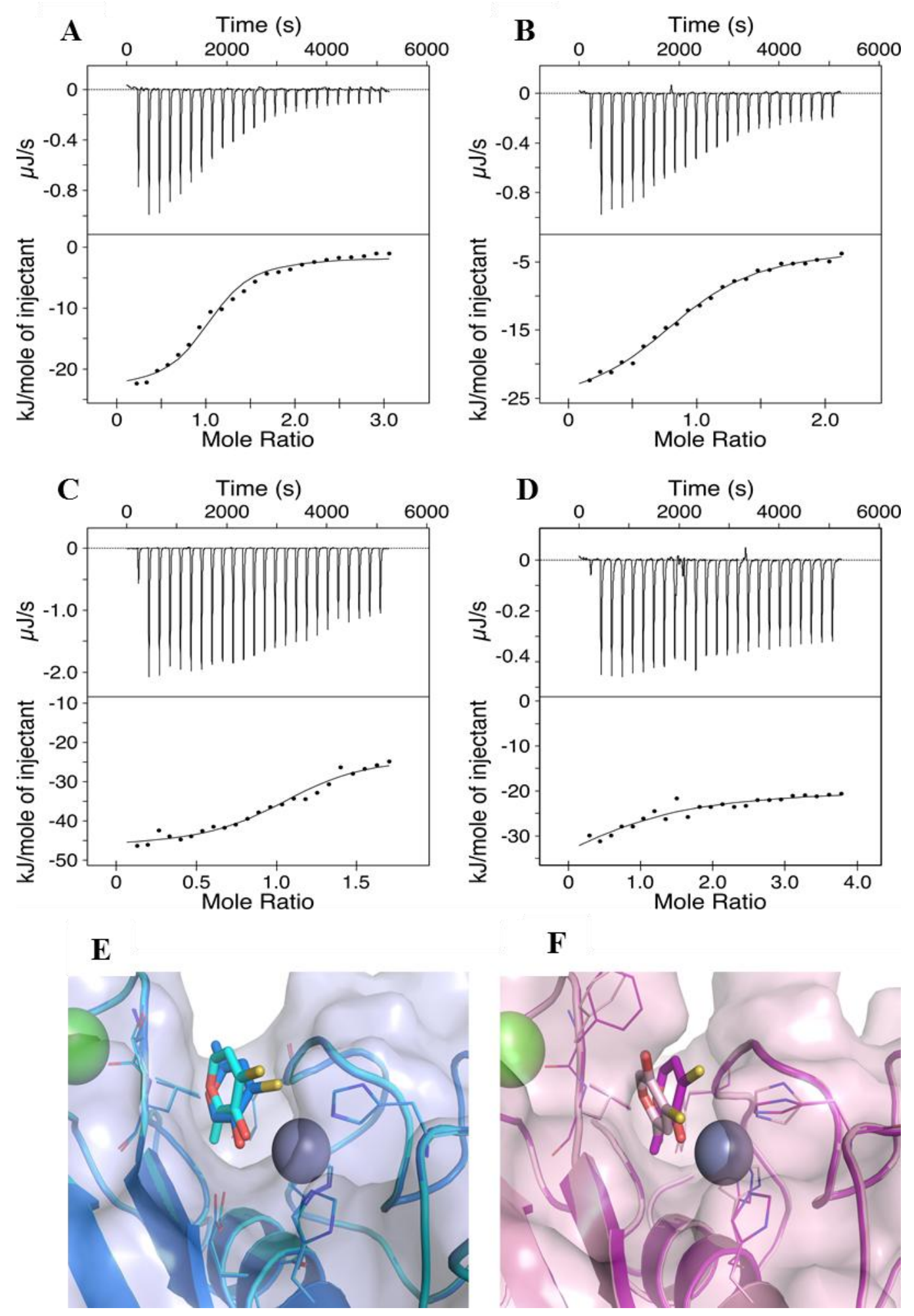

Figure 6: ITC results of ZBG binding to cdMMP7 and cdMMP16. Titration of TM (A) and

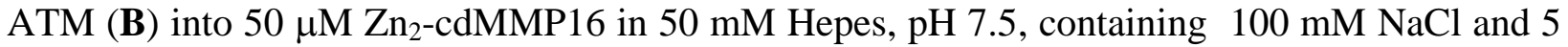




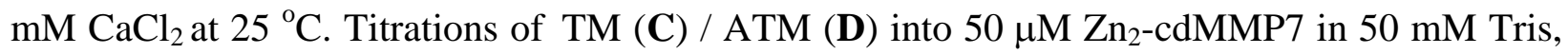
pH 7.5, containing $5 \mathrm{mM} \mathrm{CaCl}_{2}$ and $0.1 \%$ Brij 35 at $25{ }^{\circ} \mathrm{C}$. Representative binding modes from docking simulations (E) and (F). RosettaDock simulations of cdMMP7 (E) with TM (cyan) and ATM (blue). RosettaDock simulations of cdMMP16 (F) with TM (purple) and ATM (pink). Throughout, protein backbones are show as cartoons, relevant interacting side chains shown as lines, bound ions shown as spheres, and TM and ATM are shown as sticks. 